\title{
UNA LECTURA INTEGRAL DE REPÚBLICAY EL TIMEODE PLATÓN. SOBRE LA POSIBILIDAD DE UN ESTADIO BASAL DEL PENSAMIENTO POLÍTICO PLATÓNICO
}

\author{
Rodrigo Illarraga \\ Universidad de Buenos Aires
}

\begin{abstract}
A particularly difficult issue when reading the corpus Platonicum is the platonic political thought. Whithin the multiple facets, which this tematic area contains, a quite important one is the reading of different platonic texts, which seem to be referring to each other. Among these, Timaeus-Critias and Republic are remarkable (both because of their size and importance). The aim of this work ir to propose a possible interpretation of the clear resemblences between the first section of Timaeus (17c1-19b1) and certain ideas/passages developed in Republic II, III and V. Briefly, in the selected texts are presented pragmatic elements for the configuration of and ideal city, which specify social and educational patterns. This article proposes the existence of a foundational stage in the platonic political thought. It also copes with positions, which try to explain the detected resemblances through a dramatic chronology of the dialogues, and the hypothesis of a proto-Republic, which maintains an edition prior to the actual. Summarizing, this article maintains, keeping the selected sourced by the proto-Republic hypothesis (Aristophanes' Assemblywomen, Isocrates' Busiris, among others), that the interaction between Republic and the Timaeus-Critias is determined by a basic substrate of political proposals -part of te platonic thought- conceived prior to Republic and held, at least, until the composition of Timaeus-Critias.
\end{abstract}

Keywords: foundational stage, intertextualy, platonic political thought.

Resumo: Um problema particularmente dificil em torno da leitura do platonicum corpus é o pensamento político de Platão . Entre as muitas facetas que contém essa área temática, um foco emergente é a leitura dos diferentes textos de Platão em relação a temas político fazendo referência uns aos outros. Entre esses textos destacam, pela sua dimensão e sua importância no trabalho total de Platão, a República e Timeu-Crítias. O objectivo deste trabalho é encontrar uma interpretação possível das semelhanças evidentes entre a primeira seção do Timeu (17c1-19b1) e itens desenvolvidos nos livros II, III e V da República. 
Resumidamente, ambas seções delinean os elementos pragmáticos da configuração de uma cidade ideal: os sistemas que compõem os padrões sociais e educacionais. Contrariamente às posições que procuram explicar as semelhanças por meio da continuidade dramática dos dois diálogos e desafiando a hipótese de Proto-República, que suporta uma edição anterior a preservada agora, este trabalho propõe a existência de um estrato basal de pensamento político platônico. Em suma, esta abordagem suporta, mantendo as fontes selecionadas para a hipótese Proto-República (As Mulheres na Assembleia de Aristófanes, Busiris de Isócrates), que a interação entre a República eo Timeu-Critias que se baseava em propostas políticas fundamentais que percorrem a obra de Platão, foi concebida antes da República e mantida até a composição do Timeu-Critias.

Palavras-chave: etapa fundacional, intertextualidade, pensamento político platônico.

El Timeo comienza con la recapitulación de una conversación mantenida el día anterior al que se desarrolla el diálogo. En ella Sócrates ha expuesto su opinión peri politeias, intentando encontrar la mejor organización político-social posible. En este trabajo sostendremos que esta conversación mencionada, junto con su puesta en narración desarrollada en el mito de la Atlántida, constituye un elemento para habilitar y reconstruir una base del pensamiento político platónico de largo plazo, inserto asimismo en los libros II a V de República: un estadio basal que conforma el sustrato de las reflexiones sobre los elementos de la praxis organizativa de la sociedad ideal.

Para sostener esta hipótesis hemos dispuesto el siguiente recorrido. En primer lugar, abordaremos una serie de cuestiones preliminares problemáticas que traen aparejada la lectura combinada de la República y el Timeo. Estas precisiones giran en rededor de la necesidad de escindir conceptos útiles a nuestro análisis que se encuentran fuertemente ligados: la noción de proto-República, la posibilidad de un momento pre-República del pensamiento ético-político-social de Platón, y la cuestión de la unidad de esta obra. A su vez, presentaremos el concepto de estadio basal del pensamiento político platónico. En segundo lugar, analizaremos las posibilidades de enlazar dramáticamente los dos diálogos trabajados, posición mantenida por un nutrido grupo de académico. Esta hipótesis, en tanto sostiene una interrelación dramática directa entre la República y el Timeo, suponiendo así el nexo teórico inmediato entre ambos diálogos, 
atenta directamente contra el concepto de estadio basal, que se plantea como un lugar común -unificador y articulador - entre las ideas de la práctica organizativa de la sociedad de República y la de Timeo. Revisaremos el concepto de proto-República como aparato interpretativo para la situación delineada en el segundo punto. La hipótesis de protoRepública, al escindir de República concretamente la parte coincidente con Timeo, otorgándole cierta unidad editorial, se postula como un posible lugar común entre República y el Timeo. Marcaremos sus falencias teóricas y testimoniales, proponiendo la alternativa de estadio basal. En tercer lugar, expondremos los parágrafos de los diálogos en donde se evidencian las coincidencias entre los programas de las partes seleccionadas de Timeo y República, analizándolos. Finalmente, cerraremos el trabajo con las conclusiones alcanzadas.

Para abordar nuestro tema es preciso esclarecer antes una serie de elementos en los que nos apoyaremos para realizar el análisis. Para llevar a cabo el programa propuesto, debemos revisar conjuntamente el discurso recapitulador del Timeo y aquellas secciones de República que parecen aludidas en él. Como este cotejo se refiere a una parte específica del último diálogo, esta metodología trae aparejada una serie de problemáticas distintas pero fuertemente interrelacionadas y en tensión que aparecerán en el presente trabajo puestas en discusión o recapituladas brevemente.

La temática analizada en este estudio se enfrenta directamente con la hipótesis de una proto-Républica. Este constructo de Holger Thesleff ${ }^{1}$, sostenido mediante diversas fuentes textuales, se plantea como una edición anterior a la conocida hoy del diálogo platónico, que habría constituido una primera circulación del texto, distribuido en los ámbitos intelectuales de la Atenas contemporánea a Platón. Esta primera República, sostiene Thesleff, se encontraría conformada por los libros II, III y V. De este modo, la actual República habría estado redactada ensamblando en un volumen un diálogo

1 THESLEFF, 1982, THESLEFF, 1989, THESLEFF, 1997. Ver también NAILS, 1995. 
de juventud (el conjetural Trasímaco) a guisa de libro I, la proto-República, y los libros $\mathrm{V}$ al X.

Este planteo establece una discusión con una problemática harto recorrida, la de la unidad de República. El problema de la construcción del diálogo se ha centrado en la relación entre el Libro I y el resto de la obra. Desde que en el primer libro de República Platón utiliza motivos y formas propias de los llamados diálogos de juventud, ciertos autores, siguiendo al método estilométrico ${ }^{2}$, como Friedländer ${ }^{3}$ y más recientemente Thesleff, han intentado escindir esta sección del resto del texto, proponiendo que Platón habría utilizado como introducción a República un diálogo anterior no publicado por separado. Entre otros, autores como Reeve ${ }^{4}$ y Kahn ${ }^{5}$ han descartado estas hipótesis, sosteniendo que la actual estructura del diálogo tiene una razón de ser interna a la obra.

Manteniendo la línea que sostiene la unidad de República y sin desconocer ciertos testimonios textuales de la hipótesis de proto-República, nuestra propuesta es la de identificar un estadio basal del pensamiento platónico. Con este concepto pretendemos hacer referencia a un conjunto de planteos políticos presentes en gran parte de la obra de Platón. Un sustrato elemental, un denominador común, que se encuentra como base de los desarrollos relacionados con la organización política. Teniendo en vista la existencia de elementos constantes en el pensamiento político platónico nos es posible articular las similitudes entre Asambleístas de Aristófanes ${ }^{6}$ y del

\footnotetext{
2 El método estilométrico propone, sintéticamente, organizar cronológicamente la producción de un autor en base a variaciones conscientes e inconscientes en la forma de redacción. Para el trabajo inicial de esta metodología ver LUTOSLAWSKI, W, 1897, que desarrolla las líneas trazadas en CAMPBELL, 1867). Para un trabajo de estilometría contemporáneo ver LEDGER, 1989. Para una revisión general de la evolución del método estilométrico ver BRANDWOOD, 1990.

3 FRIEDLÄNDER, 1958-1969, 50 y ss.

4 REEVE, 2006, 22-24.

5 KAHN, 1993.

6 Las semejanzas entre República y Asambleístas, si bien notada por varios autores, ha sido analizada por (THESLEFF, 1997), en su propuesta de proto-República. Thesleff sostiene que esta primera edición del diálogo tuvo una importante difusión, siendo parodiada en la comedia de Aristófanes. Cf. (SEGAL, 1996), que sostiene que es Platón el que parodia a la obra en República. Estas diversas hipótesis surgen en rededor de las complicaciones que trae la compatibilización de las cronologías de redacción. A grandes rasgos, Asambleístas de Aristófanes es anterior a la composición de República. A nuestro ver no puede ser un producto de la burla hacia la obra del comediógrafo: es la comedia la que surge como construcción lúdica de una situación extraña, y no la filosofía. En ese sentido, seguimos la hipótesis de Thesleff, manteniendo que Aristófanes es impulsado a ridiculizar el pensamiento platónico. No obstante, como desarrollaremos a lo largo de este trabajo, no compartimos su propuesta en tanto sostiene que
} 
Busiris de Isócrates $^{7}$ con República (y el Timeo-Critias) sin apelar a instancias editoriales ad hoc. De la misma forma, esta herramienta teórica permite una mayor comprensión de las referencias intertexuales a elementos de República (nuevamente, posteriormente presentes en el Timeo-Critias) presentes en fragmentos de Aristipo $^{8}$ y en parágrafos Jenofonte 9 . Finalmente, permite estudiar desde una perspectiva renovada el objeto de este trabajo: las evidentes similitudes entre República y el Timeo-Critias.

Para sostener esta hipótesis, hemos de atravesar una serie de instancias de análisis concatenadas. Sintéticamente, la defensa del concepto de estadio basal aplicada a Timeo y a República precisa centrar la atención en aquellas posturas que plantean la unidad entre estos dos diálogos. Así, nuestro objetivo será, en la primera parte del presente trabajo, revisar las posiciones respecto de las diferentes formas de interacción directa pensadas para las obras analizadas: por un lado, la continuidad dramática RepúblicaTimeo, por el otro, la relación entre una hipotética proto-República y el Timeo. Ambas posturas, como hemos expresado, se nos presentan como deficientes por diversas razones para explicar las similitudes existentes: las dos posturas precisan de supuestos no habilitados (o habilitados tenuemente) por la evidencia textual.

Nuestro concepto de estadio basal del pensamiento político platónico no presenta, desde nuestra perspectiva, estas falencias: diferentes fuentes, tanto pertenecientes al corpus de Platón como externas a él, muestran el fuerte interés del filósofo en cuestiones tocantes a lo político, y

está parodia fue generada a partir de una edición material de República. Sostenemos, como se ha dicho, que el material generador de esta comedia fue lo que denominamos estadio basal del pensamiento político platónico, gestado en primera instancia de manera oral y luego puesto en texto en República y posteriormente recapitulado en el Timeo. Sin entrar en la discusión puntual, cabe resaltar que esta existencia oral, dialógica, del estadio basal, no está relacionada ni mantiene las llamadas ágrapha dógmata. En rigor del lenguaje, estas doctrinas hipotéticas son no-escritas; al contrario, el estadio basal tiene un claro apoyo textual en República y el Timeo, objeto de estudio en este trabajo.

7 Es notable la falta de trabajos sobre este texto. Existe una sección dedicada a las conexiones con República en la reciente primer edición anotada del texto (LIVINGSTONE, 2001). Otro análisis en (ILLARRAGA, 2009).

8 Notables son las conexiones entre lo puesto en boca de Aristipo en Jenofonte, Memorabilia, III.8.1-17 (= SSR, IV.A.165, en (GIANNANTONI, 1990), extremadamente semejantes a las posturas hedonistas repuestas a principios de República IV, entre otros pasajes. Actualmente nos encontramos en proceso de redacción de un artículo sobre estos temas de los que no existe, hasta donde llega nuestro conocimiento, material específico.

9 Para las conexiones con Ciropedia ver (NADON, 1996). Para las presentes en Anábasis ver (HOWLAND, 2000). 
en particular, al aparato político como rector de la sociedad. Asimismo, estos testimonios muestran la profunda interrelación de las propuestas políticas platónicas resaltadas a lo largo del trabajo con otras presentes en las obras de intelectuales contemporáneos a Platón. Por otro lado, la propuesta de un estadio basal nos permite una intelección económica de la aparente contradicción presente en la lectura comparada de República y del Timeo: por un lado, similitudes teóricas evidentes, por el otro, incompatibilidad dramática.

En resumen, las claras semejanzas semánticas y lingüísticas en torno a la recapitulación del discurso socrático del día anterior y los primeros elementos del mito de la Atlántida (Tim. 17c1-19e2) y parágrafos de los libros II, III y V de República responden a un sustrato común, un estadio basal, del pensamiento político platónico, presente en Republica, desarrollado en ella, y perviviente, al menos, hasta la redacción del Timeo, en donde es nuevamente expuesto en forma resumida.

\section{II}

En primer lugar, debemos analizar la posibilidad de plantearse si cabe enlazar dramáticamente República y Timeo tal y como han llegado a nuestros días, esto es, si ambos textos se encuentran ubicados consecutivamente a nivel dramático en la cronología literaria propuesta por Platón. De ser posible ubicar República inmediatamente antes que el Timeo, dramáticamente hablando, la pregunta que nos tendríamos que hacer es, como plantea Taylor ${ }^{10}$, sobre la razón por la cual ciertos elementos del primer diálogo fueron elididos en el segundo.

Confeccionando un mínimo estado de la cuestión es posible conformar el siguiente esquema de posturas con respecto a la datación dramática de República:

I) Un grupo que localiza la acción de República circa 410/11 a.C.: Campbell y Jowett ${ }^{11}$, Shorey ${ }^{12}$, Adam ${ }^{13}$.

10 TAYLOR, 1927.

11 CAMPBELL, JOWETT, , 1894, 3.2: 410/11 a.C. 
II) Un grupo que localiza la acción de República circa 420/21 a.C.: Bloom $^{14}$, Taylor ${ }^{15}$, Howland ${ }^{16}$.

Trayendo a colación estas posturas, y otras que hemos mencionado con antelación, Debra Nails concluye sintéticamente:

If the Republic sprang whole from the head of Plato, no univocal dramatic date would thereby be entailed, but the expectation would gain rather in credibility. If not, if Plato wrote a proto-Republic before the Academy was established, then its existence militates against the possibility of determining a single dramatic date for the dialogue. And if the Republic was stitched together from a separate Book I, a proto-Republic, and new material, and revised late into an almost seamless whole, then a single firm dramatic date seems ever more implausible. ${ }^{17}$

Con respecto al Timeo, Lampert y Planeaux realizan un exhaustivo trabajo en donde, mediante el cruce con fuentes históricas, concluyen que la fecha dramática del diálogo se ubica en el verano de 421 a.C. ${ }^{18}$, ubicación que concuerda con la postulada por Taylor ${ }^{19}$.

Ahora, por más atractiva que resulte la propuesta de República abriendo una serie de diálogos que continuaría con el Timeo desde el punto de vista teórico, en tanto es posible establecer hilos conductores transtextuales, lo cierto es que existe una serie de puntos insalvables para observar una plena identificación entre ambas obras del corpus, aún habilitando la lectura separada de ciertas partes de República. Por un lado, el pasaje recapitulativo del Timeo $(17 \mathrm{c} 1-19 \mathrm{~b} 1)$ cierra con la afirmación taxativa de que nada de lo que se expuso el día anterior ha quedado fuera del resumen hecho; en tanto República tiene en sí más contenido que el

12 SHOREY, 1937, introduction): $410 / 411$ a.C.

13 ADAM, 1902: tentativamente 409 a.C.

14 BLOOM, 1968, 440, n.3: 420/421 a.C.

15 TAYLOR, 1955, 263-264: 422/421 a.C.

16 HOWLAND, 2004, XII: 420/421 a.C.

17 NAILS, 1998, 39.

18 LAMPERT, PLANEAUX, 1998.

19 TAYLOR, 1927, 17. Cf. (CORNFORD, 1997, 5) 
expuesto en los puntos de la recapitulación, la identificación pierde fertilidad.

Sócrates: ¿Podemos decir entonces que hemos ido a través del discurso de ayer (khthés), en tanto es un requisito para una revisión sumaria (hós en kephalaíois pálin epanelhein), o hay algún punto omitido (apoleipómenon), querido Timeo, que debamos ver agregado?

Timeo: En absoluto, esto era lo que ayer dijimos, Sócrates (oudamós, allà autà taût' hèn tà lekhthénta, ho Socrátes). (Tim. 19a7-b1) ${ }^{20}$

Por otro lado, los personajes que participan en la conversación al día previo al Timeo son Hermócrates, Timeo, Critias, Sócrates y un personaje ausente en el presente del diálogo y cuyo nombre no es explicitado, protagonistas que se caracterizan, como marcan Lampert y Planeaux, por poseer experiencia y predisposición tanto hacia el ejercicio filosófico como político; ahora, en República los interlocutores son, principalmente (esto es, teniendo en cuenta la dinámica narrada a partir del libro II), Sócrates, Adimanto y Glaucón: dos jóvenes ansiosos, muy diferentes que los experimentados hombres del Timeo $^{21}$. Finalmente, en el nivel de cronología dramática resulta extremadamente dificultoso compatibilizar las fechas y datos presentes en ambos textos, aún aceptando que los dos diálogos tienen como objetivo literario representarse dentro de una realidad históricocontextual determinada, asunción no compartida por todo el mundo académico $^{22}$.

En las últimas décadas del siglo XIX y las primeras del siglo XX una serie de autores ${ }^{23}$ propusieron, basándose -entre otras fuentes- en el pasaje

20 Para el texto traducido del Timeo seguimos LISI, 1992, con variaciones propias guiados por LAMB, 1925) y BURNET, 1900-1907. Se han consultado asimismo las ediciones de CORNFORD, 1997 y TAYLOR, 1927.

21 Aún ampliando el repertorio de interlocutores y personajes, incorporando a Trasímaco, Céfalo, Lisias, Polemarco, Eutidemo, Carmántides, Nicerato y Clitofonte, República sigue mostrándose completamente distinta al Timeo en lo que refiere a esta diagramación.

22 Para posturas que propongan la falta de interés de parte de Platón por reponer una realidad histórica dramática ver, para República, MOORS, 1987; para Timeo, CORNFORD, 1997. Ver supra n.20.

23 Entre otros, POST, 1927. 
del Timeo que se extiende de 17c1 a 19b1, que la República actual es una segunda edición del diálogo. En los últimos veinte años una serie de trabajos, cuya autoría corresponde mayoritariamente a Thesleff, han revivido esta noción bajo el nombre de proto-República ${ }^{24}$. El concepto es que existió una primera edición de República, compuesta por los libros II, III y $\mathrm{V}$, previa a la conformación de la Academia, que habría sido ampliada y revisada para la redacción definitiva que ha llegado a nuestros días, movido Platón por la mala recepción de su primera versión.

Esta hipótesis se apoya tanto en una vaga referencia de Aulo Gelio en Noches Áticas ${ }^{25}$, muy posterior al siglo IV a.C., como en las intertextualidades o alusiones presentes en textos de contemporáneos a Platón. La principal de estas es Asambleístas de Aristófanes (obra anterior a la redacción de República), donde se traza la parodia de un sistema político muy similar a los puntos centrales de los libros II, III y $\mathrm{V}^{26}$. Por otro lado, ciertos elementos característicos referidos a la organización social en clases o estratos, presentes en estos libros, también son encontrados en el discurso encomiástico Busiris, de Isócrates $^{27}$. Finalmente, Thesleff ${ }^{28}$ y Nails ${ }^{29}$ marcan como otro argumento sugerente la recapitulación del discurso socrático peri politeias que se encuentra en la apertura del Timeo.

En el caso de conceder que la acción del Timeo se sitúa en el año 421 a.C., podría fácilmente caerse en la tentación, siguiendo a Thesleff y Nails, de apoyar una proto-República: escindiendo los libros II, III y V y ubicando su fecha dramática en 420/421 a.C. (cosa imposible para la totalidad de

\footnotetext{
24 En primer lugar, THESLEFF, 1982 y la posterior revisión de este trabajo, THESLEFF, 1989. Para ver un trabajo especialmente dedicado al concepto de proto-República, THESLEFF, 1997.

25 "quod Xenophon inclito illi operi Platonis, quod de optimo statu reipublicae civitatisque administrandae scriptum est, lectis ex eo duobus fere libris qui primi in volgus exierant, opposuit contra conscripsitque diversum regiae administrationis genus" // "Jenofonte, en contra a ese celebrado trabajo de Platón, en donde esta escrito sobre la mejor forma de constitución y de administrar un estado, habiendo apenas leído los primeros dos libros del trabajo de Platón que se hicieron públicos primero, propuso un modo diferente de gobierno." (N.A., XIV, III.3) La traducción es nuestra siguiendo la edición y traducción de ROLFE, 1927.

26 Este punto esta especialmente tratado en THESLEFF, 1997. Cf. TAYLOR, 1955, 264 y SEGAL, 1996, 321.

27 Ver LIVINGSTONE, 2001.

28 THESLEFF, 1989, 11.

29 NAILS, 1998, 394.
} 
República según Nails $^{30}$ ), existiría un diálogo hoy perdido que sería ese discurso peri politeias llevado a cabo el día anterior a la acción del Timeo.

Este planteo suscita una serie de problemáticas. En primer lugar, dividir el libro I, los libros II-V y los libros V-X, rompiendo la totalidad que constituye República significa desconocer la unidad que tiene el diálogo en cuanto a temáticas, el progresivo tratamiento de los mismos tópicos conforme avanza el desarrollo del texto, y el eje literario común que lo atraviesa $^{31}$. En segundo lugar, es imaginar que Platón es presa de su obra, que para crear una situación ficcional en donde se plantee una propuesta teórico-filosófica necesitase tenerla primero en diálogo. En otras palabras, que Platón precisa, para poder describir una conversación imaginaria en un diálogo, haberla volcado previamente en un texto material. Esta suposición no tiene asidero en toda la obra platónica, donde se reproducen o mencionan diálogos mantenidos por personajes en otro momento sin existir evidencia textual de su registro. En tercer lugar, es acusada la manifiesta falta de testimonios que avalen el de Aulo Gelio: no existe otro texto o fragmento que habilite explícitamente la Proto-República, mientras que sí existen, de hecho, fuentes que proponen configuraciones del modo de escritura platónico completamente diferentes, como el de escritura continua que Howland encuentra en pasajes de Aristóteles y Dionisio de Halicarnaso ${ }^{32}$.

Desde nuestra perspectiva estos obstáculos son infranqueables: aceptar la proto-República implica comprometerse con una serie de principios metodológicos, como mínimo, poco fructíferos ${ }^{33}$.

\section{Ver supra, n.17}

31 Como ya se ha anotado (ver supra, n.4 y 5), un ejemplo de trabajo que propone la cohesión del diálogo, en contra de las posturas que intentan verlo como una suma de escritos redactados en diferente momento es el de (KAHN, 1991). referido a la unidad existente entre el libro I y el resto de República, donde se propone que el primer libro del diálogo repone una forma tradicional de los textos aporéticos de juventud para presentarse como una suerte de preludio que resuma la línea de trabajo del diálogo.

32 HOWLAND, 1991, 200-201. La hipótesis de la "revisión continua" sostiene que Platón revisó sus textos a lo largo de la vida, postura que invalida no solo la posición de Thesleff, sino también al método estilométrico, que sostiene marcados momentos diferenciados de producción textual. Trayendo a colación el modelo de Howland no queremos sostenerlo, sino marcar los diferentes posibles modelos de escritura que son habilitados por las fuentes existentes.

33 Por un lado, los ya mencionados, (I) diálogos dispuestos a ser indiscriminadamente divididos, (II) incapacidad por parte de Platón de narrar situaciones ficcionales sin una previa primer puesta en texto, (III) aprobación plena de un testimonio aislado imposible de ser constatado. Por otro lado, la hipótesis de proto-República se propone como una primera instancia editorial, por lo que ubica al foco de análisis en la existencia material de un texto anterior a la misma República. En primer lugar, comprobar la existencia de un texto hipotético del que no hay prueba alguna es imposible, por lo que se convierte en una tarea 
No obstante, si bien se nos aparece como imposible salvar en concreto la totalidad de hipótesis sostenidas por Thesleff, creemos posible rescatar cierta idea presente en ella: existe, de hecho, un momento de configuración del pensamiento ético-político platónico anterior a la República -un estadio basal en nuestras palabras- cuyo núcleo, las propuestas más pragmáticas, se encuentra en los libros II a $\mathrm{V}$ de este diálogo.

Sostenemos que la evidencia textual presentada para refrendar la hipótesis de proto-República debe dejar de ser articulada por el testimonio de Aulo Gelio, y debe encontrarse otro eje de análisis. Los testimonios disponibles en fuentes paralelas, sin la cita en extremo tardía de Noches Áticas, habilitan la posibilidad de encontrar la configuración del estadio basal del pensamiento político de Platón en tensión con las posturas de intelectuales que le son contemporáneos ${ }^{34}$, sin que sea preciso abordar las cuestiones referidas a la edición del texto, imposibles de refrendar con los testimonios disponibles.

Así, nuestra propuesta consiste en analizar sintéticamente una sola de las facetas disponibles; en otras palabras, como ya hemos adelantado, nuestra intención es mostrar cómo el Timeo-Critias puede ser utilizado para acompañar el trazado de esta base política presente en la filosofía de Platón.

Mantenemos que Platón configuró este sustrato teórico referido a lo político con antelación a República, y que es de este estadio basal de su pensamiento político del que abreva para narrar el discurso socrático peri politeias del día anterior al Timeo. En otras palabras, en el Timeo Platón no hace referencia a República (o a Proto-República) -ya hemos expuesto argumentos para mostrar la imposibilidad de esto-, sino que tanto en

fútil; en segundo lugar, su existencia o inexistencia no modificaría el esquema del pensamiento platónico, en tanto ese texto primigenio se encontraría inserto en la actual República y, en menor medida, en el Timeo. En tanto la propuesta de proto-República y sus principios metodológicos nos embarcan en una agenda imposible de constatar y carente de frutos para el análisis del pensamiento de Platón, se nos presenta como una aproximación carente de resultados.

34 Para el concepto de zona de tensión dialógica, que prefigura un área de discusión en un grupo amplio de intelectuales en torno a tópicos comunes, en donde las diferentes posiciones argumentativas generan cambios en las diferentes teorizaciones ver MÁRSICO, 2010. Para una distribución prosopográfica de los intelectuales en torno a Platón ver NAILS, 2002. Los testimonios y fuentes paralelas referidos que fortalecen la hipótesis de un estadio basal del pensamiento político platónico no son objeto de este trabajo, no obstante, han sido mencionados en la nota 6 . 
República como en el Timeo se está observando un mismo programa ideal político, común a las dos obras ${ }^{35}$.

\section{III}

Al comienzo del Timeo, precisamente desde 17c1 a 19b1, Sócrates se propone reconstruir de manera resumida lo expuesto el día anterior a pedido de Timeo. Lo dialogado pretéritamente ha tratado, según lo dicho, sobre cuál sería el mejor ordenamiento político posible y sobre qué hombres podrían llevarlo a cabo. En rigor, este proyecto tiene una serie de puntos claramente distinguibles:

1) División en clases, separando a campesinos (georgôn) y demás artesanos de los guerreros, quienes defienden la ciudad (propolemesónton). (Tim.17c4-8)

2) Dirección de las funciones en concordia con la naturaleza (phýsis) propia de cada individuo, siguiendo el principio de especialización que propone centrar los esfuerzos del sujeto alrededor de una única técnica (tékhne). (Tim.17c9-d1)

3) Doble carácter anímico de los guardianes (phylákes), suaves (práos) y fieros (khalepós), dependiendo de que traten con amigos (philois) o con enemigos (ekhthrón). (Tim.17d3-18a2)

4) Importancia de una educación conjunta (koinè paideía) completa de acuerdo a criterios previamente establecidos para la clase de los guardianes (Tim.18a8-10)

35 Esta hipótesis de un pensamiento político básico y permanente a lo largo de su devenir intelectual permite entender que el tratamiento hecho por Aristóteles en Política a Platón esté referido primordialmente a ciertos puntos de República, los mismos que coinciden mencionados en Timeo, antes que a las Leyes. 
5) Guardianes desposeídos de riqueza (ktéma) y propiedad, dependientes del común de la sociedad para su manutención, en forma de salario (misthós). (Tim.18b1-7)

6) Otorgar a los guardianes mujeres (gynaîkes) de naturalezas similares (paraplesíai phýseis) a ellos, y asignarles las mismas actividades. (Tim.18c1-4)

7) Comunión tanto de matrimonios como de familias, estableciendo una política sexual articulada en torno a la manipulación secreta por parte de los gobernantes. (Tim.18c6-d4).

8) Crianza y supervisión de los infantes (paîdes), a fin de seleccionar a los mejores (hoi agathoí). (Tim.19a1-5)

Las características enunciadas detallan en su completitud, según explicita Timeo tras una pregunta ad hoc de Sócrates, el panorama del sistema propuesto en la discusión sucedida el día anterior al presente dramático.

A su vez, a pedido de Sócrates, que desea ver su sistema en movimiento, esto se inserta en una historia: se narra el mito de la Atlántida, el sucinto resumen de una guerra que involucra a una sociedad que repite el modelo socrático. Estos pasajes comprenden los siguientes puntos:

1) Creación de un relato construido ad hoc, que corresponde al sistema propuesto por Sócrates, esto es, en concordia con propuestas o verdades filosóficamente analizadas. (Tim.19c2-e2)

2) Construcción de un andamiaje mítico que legitima el relato, mostrándolo como inserto en un pasado remoto e inescrutable, sustentado mediante referencias comunes a la mentalidad griega dispuestas para fundar en la antigüedad la veracidad de lo presentado. (Tim. 21a7-27b6)

Estos pasajes que resaltamos, tanto los que refieren a la recapitulación del discurso socrático como los que tratan del mito de la Atlántida, han llamado poderosamente la atención de numerosos 
académicos: el diálogo descrito mantenido con antelación al desarrollo del Timeo-Critias entre Sócrates y sus interlocutores tiene acusados puntos en común con República, lo que ha hecho pensar en una posible conexión dramática entre ambos textos. Dos comentaristas ya clásicos pueden dar cuenta de las posturas asumidas. Por un lado, Taylor propone que las características del sistema social desarrollado son una clara alusión a República: si algo falta en este resumen (como por ejemplo, la existencia del filósofo-rey, entre otras cosas) -propone Taylor-, debemos preguntarnos el motivo de esta ausencia ${ }^{36}$.

Cornford, por otro lado, postula una mirada diferente:

Plato could not have stated more plainly that Socrates is not to be supposed to have narrated the whole conversation in the Republic as we have it. It follows at once that he did not intend the Republic to stand as the first dialogue in his new series [...] No doubt Plato was thinking of the contents of that part of the Republic and intending his readers to recall them; but he was not the slave of his own fictions. ${ }^{37}$

Estas dos posiciones se han constituido en punto de partida para los estudios del Timeo, y la asunción de alguna de ellas, no siempre de manera argumentada y explícita, guía la revisión del diálogo.

Veamos en detalle cuáles son los puntos de este programa presentes coincidentemente de manera literal en la recapitulación del discurso peri

36 TAYLOR, 1927, 4. Bajo esta perspectiva se enmarca, por ejemplo, la pregunta por la ausencia de "filósofos-reyes" en la recapitulación del Timeo hecha por (SCHOFIELD, 1999, cáp.2). Adelantando nuestra perspectiva, que configura un estadio basal que está plenamente expresado en Timeo y en secciones de la República, antes que preguntarnos por la falta de "filósofos-reyes" en el Timeo, nos preguntamos por su presencia en República.

37 CORNFORD, 1997, 4. 
politeias del Timeo y República, guiándonos con la lista de ocho puntos que hemos propuesto.

$\begin{array}{ll}\text { 1. División en } & \text { "Sócrates: ¿No fue acaso } \\ \text { clases, separando a } & \text { nuestra primera medida } \\ \text { campesinos y } & \text { separar en ella a los } \\ \text { demás artesanos } & \text { campesinos (georgôn) y a } \\ \text { de los guerreros, } & \text { los otros artesanos del } \\ \text { quienes defienden } & \text { estamento de los que } \\ \text { la ciudad. } & \text { luchan en defensa de ellos } \\ & \text { (propolemesónton)? } \\ & \text { Timeo: Si" } \\ & \text { (Tim.17c4-8) }\end{array}$

\begin{abstract}
"Asimismo, las tareas concernientes a la guerra, ¿no es crucial que estén bien realizadas? ¿O es tan fácil ser soldado (polemikós) que incluso cualquier campesino (georgôn) o zapatero podrá serlo y seguir cumpliendo al mismo tiempo con su otro oficio, mientras que nadie podría llegar a ser buen jugador de damas o dados si no lo practicara desde niño, sino que lo hiciera como algo ocasional? Y si toma el escudo o alguna otra de las armas de guerra y herramientas, ¿podrá ser en el mismo día un soldado de infantería (hoplitikês) o un combatiente de otro tipo de lucha militar, mientras que tras haber tomado las herramientas de otros oficios no podrá convertirse ni en un artesano (demiourgón) ni en un atleta, y tampoco este tipo de herramientas será de utilidad al que no tenga el conocimiento de cada una ni haya tenido una práctica suficiente?
\end{abstract}

(Rep., II.374c1-d6) 1

En ambas obras se plantea la necesidad de dividir la sociedad en función de las actividades militares. Se escinde por un lado a los militares (propolemoûntes, hoplítes) del pueblo campesino (georgoî) y artesano (demiourgós), en vistas de la búsqueda por una mayor pericia.

1 Para el texto traducido de República seguimos principalmente a MÁRSICO, DIVENOSA, 2005), con variaciones propias contemplando CAMPBELL, JOWETT, 1894, ADAM, 1902, BURNET, 1900-1907 y SHOREY, 1937. 
2. Dirección de las funciones en concordia con la naturaleza propia de cada individuo, siguiendo el principio de especialización que propone centrar los esfuerzos del sujeto hacia una única técnica.
"Sócrates: Y luego de asignar a cada uno una ocupación única para la que estaba naturalmente (phýsin) dotado, una única técnica a cada cual (mían hekásto tékhne)..." (Tim.17c9-d1)
"Del mismo modo le asignamos también a cada uno de los demás una sola tarea, para la cual cada uno estaba naturalmente dotado (epephýkei hékastos), y en la cual iba a trabajar durante su vida, dispensado de las otras, pero sin desatender los momentos propicios para lograr un buen producto"

(Rep., II.374b9-c2)

La división en clases, tanto en República como en el Timeo, es sustentada en el principio de especialización y por una organización en torno a la naturaleza (phýsis) de los individuos, que configura individuos abocados a una sola técnica (tékhne), estando dispensados de otras actividades a fin de desarrollarse plenamente en una.

3. Doble
carácter anímico
de los
guardianes,
suaves y fieros,
dependiendo de
que los sujetos
tratados sean
amigos o
enemigos.

\begin{abstract}
"...Afirmamos que aquellos que tenían la misión de luchar por la comunidad deberían ser solo los guardianes (phýlakes) y que, mientras que a sus súbditos tenían que administrarles justicia con suavidad (práios), ya que son por naturaleza (phýsei) sus amigos (philois), era necesario que en las batallas fueran [sc. los guardianes] fieros (khalepoí) con los enemigos (ekhthrôn) que les salieran al paso." (Tim.17d3-18a2)
\end{abstract}

\begin{abstract}
“...es necesario que [sc. los guardianes] con los que son dueños de la casa [i.e. de la ciudad] sean suaves (práious) y en cambio fieros (khalepoús) sólo con los enemigos, pues si no, no esperarán que los otros aniquilen a los ciudadanos, sino que los matarán ellos mismos con sus acciones" (Rep., II.375c1-3)
\end{abstract}

En los dos textos se afirma que la clase guerrera o guardiana debe tener una doble predisposición anímica, dependiente del sujeto al que se dirigen: los guardianes (phýlakes) deben tratar suavemente (práios) a los conciudadanos, prestos a guardar sus intereses y protegerlos; a su vez es preciso que sean agresivos (khalepoí) con los enemigos, a fin de proteger correctamente a la ciudad (pólis) ${ }^{1}$.

1 Sobre el carácter doble que tienen que conjugar los guardianes ver SCHOFIELD, 2008. 
4. Importancia de una educación conjunta completa de acuerdo a criterios previamente establecidos para la clase de los guardianes.
"Sócrates: [...] ¿No decíamos que estaban educados en gimnasia y en música (gymnastikè kaì mousikè manthémasín), y en todas las materias convenientes para ellos?

Timeo: Por cierto." (Tim.18a8-10)
"Entonces, después de la música, los jóvenes deben ser educados en la gimnasia (metà dè mousikèn gymnastikêi threptéoi hoi neaníai)" (Rep. III.403c8)

La propuesta educativa, en ambos textos, pone énfasis en la educación tanto corporal como intelectual. Los dos campos que representan esta doble preocupación son por un lado la música (mousiké), terreno que engloba tanto la práctica instrumental como el estudio de la armonía (y mediante ella, la matemática), y por el otro, la gimnasia (gymnastiké).

5. Guardianes
desposeídos de
riqueza y propiedad,
dependientes del
común de la
sociedad para su
manutención, en
forma de salario.

"Sócrates: Y se sostuvo, creo, que los así educados no debían considerar como propios (heautôn ídion nomízein) ni el oro (khrysón) ni la plata (ágrygon) ni ninguna otra posesión (ktêma), sino que, como guardianes (phylakês), habian de recibir un salario (misthón) por la guardia de aquellos a quienes preservaban -lo suficiente para gente prudente-, y gastarlo en común (koinêi) en una vida en la que compartían todo (syndiaitoménous), ocupándose exclusivamente de cultivar la excelencia (ékhontas aretês), descargados de todas las otras actividades"

(Tim.18b1-7)
"Entonces -dije yo-, mira si deben vivir y habitar de esta manera, si es que van a ser así: primero, nadie poseerá ningún bien privado que no sea estrictamente necesario (ousian kekteménon medemían medéna idían, àn mè pâsa anánke). Luego, no habrá para ellos ninguna casa ni almacén al que no acceda todo el que quiera. En cuanto a las provisiones que necesitan, los hombres atletas de la guerra, moderados y valientes, recibirán un salario (misthón) por la custodia, pagado por los demás ciudadanos (tôn állon politôn), que no les sobre ni les falte para vivir un año." (Rep.,IIl.416d5-e3) 
La comunión de los bienes aparece en República y en el Timeo como el modo de vida determinado para los guardianes, cuya práctica debe inculcarse desde pequeños en su educación. En rigor esta situación implica el alejamiento, por parte de la clase guerrera, tanto de posesiones metálicas oro (khrysón) y plata (ágrygon)- como de cualquier otro tipo de propiedad (ktêma). Los bienes (ousíai) estrictamente indispensables para la vida de los guardianes serán poseídos comunalmente, siéndoles entregados por el común de la ciudadanía (tôn politôn) en forma de estipendio (misthón) a cambio de su función como protectores ${ }^{2}$.

$\begin{array}{lll}\begin{array}{l}\text { 6. Selección de } \\ \text { mujeres de }\end{array} & \text { "Sócrates: Además, por lo } & \text { "...hay que elegir mujeres (gynaîkes) de } \\ \text { naturaleza similar } & \text { que hace a las mujeres } & \text { este tipo [sc. que tengan aptitudes para ser } \\ \text { a los hombres } & \text { mención a que sus } & \text { guardianas] para que convivan (synoikeîn) } \\ \text { guardianes, } & \text { naturalezas (phýseis) } & \text { con este tipo de hombres y compartan con } \\ \text { destinadas a } & \text { debían estar en sintonía } & \text { (symphyláttein), puesto que son aptas y } \\ \text { matrimonio con } & \text { con hombres similares } & \text { afines a ellos en su naturaleza (tèn phýsin)" } \\ \text { ellos y a realizar } & \text { (paraplesías), y a que las } & \text { (Rep., V.456b1-3) } \\ \text { sus mismas } & \text { actividades asignadas a } & \text { "..tú, legislador, del modo en que elegiste a } \\ \text { actividades. } & \text { ellos, tanto en la guerra } & \text { los hombres, así también elegirás a las } \\ & \text { como en otras actividades } & \text { mujeres, y les darás las de naturaleza } \\ \text { de la vida, debían ser en } & \text { similar tanto como sea posible (hósper toùs } \\ \text { todo caso comunes } & \text { ándras exélexas, hoúto kaì tàs gynaîkas } \\ \text { (koiná) para todos. } & \text { ekléxas paradóseis kath' hóson oión te } \\ \text { (Tim.18c1-4) } & \text { homophyeîs). Y ellos, dado que tienen } \\ & & \text { vivienda y comida en común (koiná) y } \\ & \text { ninguno posee nada en privado, vivirán } \\ & \text { juntos y, mezclándose en los gimnasios y } \\ & & \text { otros ámbitos educativos por necesidad, } \\ & \text { creo, serán conducidos de modo natural } \\ & \text { (emphýtou) a la unión mutua" } \\ & \text { (Rep., V.458c6-d3) }\end{array}$

En ambos diálogos se afirma que existen mujeres (gynaîkes) que comparten las diversas naturalezas presentes en el género masculino y, por lo tanto, que hay mujeres que pueden ocupar el cargo de guardianas junto a

2 Para estas temáticas ver BARKER, 1918, cáp.9. 
los hombres. En el programa descrito estas "guardianas" comparten tanto las funciones como el modo de vida propio de los guardianes, llevan adelante las mismas tareas y responsabilidades que sus contrapartes masculinas (symphyláttein). Bajo lo descrito, la convivencia (synoikeîn) entre individuos de diferente sexo pero de igual naturaleza (phýsis) tiene como fin lograr uniones entre quienes poseen las mismas predisposiciones ${ }^{3}$.

7. Comunión tanto
de matrimonios
como de familias,
estableciendo una
política sexual
articulada en torno
a la manipulación
secreta por parte
de los
gobernantes.

"Sócrates: ¿Y qué hay de la procreación (paidopoiías)), ¿o la singularidad de lo dicho no hace que se recuerde fácilmente?, porque dispusimos que todos tuvieran sus matrimonios y sus hijos en común (hóti koinà tà tôn gámon kaì tà tôn paídon pâsin hápanton etíthemen), cuidando de que nunca nadie reconociera (gnósoito) como propio al engendrado por él, sino que todos consideraran a todos de la misma familia: hermanas y hermanos a los de la misma edad, a los mayores, padres y padres de sus padres y a los menores, hijos de sus hijos."

(Tim.18c6-d4)
"Que todas estas mujeres (tàs gynaîkas) de todos estos hombres deben ser comunes (pánton pásas eînai koinás) y ninguna convivirá (synoikeîn) en privado con ninguno. Asimismo los hijos también serán comunes (kaì toùs paîdas aû koinoús) y ni el padre debe conocer (eidénai) a su hijo ni el hijo a su padre" (Rep., V.457c9-d3)

“-Pero los padres y las hijas y los que
acabas de nombrar, ¿cómo se
reconocerán (diagnósontai)
mutuamente?
-No lo harán de ninguna manera
(oudamôs)-dije yo-. Por el contrario,
desde el día que uno de ellos participa
de una boda, a los niños que nazcan
diez meses después de ese día -o siete,
claro-, a todos ellos se dirigirá como
hijos, si son varones, y como hijas, sin
son niñas, y ellos a él como padre, y así
en efecto los hijos de éstos serán
llamados nietos, y éstos le llamaran a su
vez abuelo y abuela, mientras que los
nacidos en el tiempo en el cual las
madres y los padres procrearon,
hermanas y hermanos, de modo que,
como antes decíamos, no se unirán
sexualmente entre ellos."
(Rep., V.461c9-d)

3 Para el lugar de la mujer en el sistema platónico, y en particular, la situación de paridad con respecto al hombre ver SAXONHOUSE, 1976, SALKEVER, 1986, FORDE, 1997. 
Tanto en la República como en el Timeo se propone que las relaciones sociales de los guardianes deben estar organizadas en torno a la comunión, no solo de bienes (ousíai), sino también de nexos familiares. Se delinea una estructura donde, por un lado, las mujeres son comunes (koinái) a todos los hombres, no pudiendo convivir en privado (synoikeîn) con ninguno; mientras que, por el otro, no existen las familias en el sentido tradicional de la palabra: las relaciones filiares se trasladan del ámbito de lo privado al de lo común, competente a toda la sociedad, a través del manejo de la información referida a los nacimientos, impidiendo el reconocimiento (eidénai, gnósoito, diagnósontai) de las conexiones padre-hijo, para lograr una identificación general basada ya no en lo personal, sino en lo generacional $^{4}$.

\section{Crianza y supervisión de los infantes, a fin de seleccionar a los mejores.}

"Sócrates: ¿Y también deciamos
que tenían que educar a los hijos
de los mejores (tôn agathôn) y
distribuir secretamente (láthrai
diadotéon) a los de los peores
(tôn kakôn) a la otra ciudad (tèn
állen pólin) y observarlos durante
su crecimiento para hacer
regresar siempre a los que lo
merezcan (toùs axíous) y pasar
a la región de la que éstos
habían vuelto a no merecedores
(anaxíous) que se habían
quedado con ellos?
Timeo: Así es."
(Tim.19a1-5)

"-Tras hacerse cargo de ellos, llevarán a los hijos de los mejores (tôn agathôn), me parece, a una guardería junto a niñeras que habiten en una parte de la ciudad separada del resto (én tini mérei tês póleos). A los de los peores (tôn kheirónon) y a alguno de lo otros que nazca deforme los esconderán en un lugar secreto y desconocido según convenga (en aporrétoi te kaì adéloi katakpýpsousin hos prépei).

-Esta es la condición -dijopara preservar la pureza de la estirpe de los guardianes (tò génos tôn phylákon)."

(Rep., V.460c1-5)

Para preservar la correcta naturaleza del estrato de los guardianes, garantía del funcionamiento y defensa de la ciudad (pólis), se establece un 
control de la naturaleza de los niños, brindándoles educación acorde a ella. Los hijos de los mejores (tôn agathôn) serán separados de los peores (tôn kakôn), quienes se trasladarán a otra parte de la ciudad de manera subrepticia (láthrai diadotéon, en aporrétoi te kaì adéloi). Esta separación a priori contempla, no obstante, el posterior control de los niños, a fin de observar su progreso, distinguiendo entre aquellos a los merecedores (toùs axíous) de regresar a la ciudad (pólis) de aquellos que no lo merecen (anaxíous).

Por fuera de la recapitulación que tiene lugar en Timeo 17c1-19b1, pero inmediatamente después de ella, se extiende una versión sintética del mito de la Atlántida, tema central del inconcluso diálogo posterior al Timeo, el Critias. La historia relatada por Critias en el Timeo tiene plena conexión con la recapitulación llevada a cabo, pues se desarrolla por el pedido de Sócrates de ver en acción lo prefigurado el día anterior al presente dramático del diálogo.

Sócrates: Quizás queráis escuchar ahora lo que me sucede con la continuación de la historia de la república (tês politeías) que hemos descrito (diélthomen). Creo que lo que me pasa es algo así como si alguien, después de observar bellos animales (zôia kalá), ya sea pintados en un cuadro o realmente vivos pero en descanso, fuera asaltado por el deseo de verlos moverse (kinoúmena) y hacer, en un certamen, algo de lo que parece corresponder a sus cuerpos. Lo mismo me sucede respecto de la ciudad (tèn pólin) que hemos delineado. Pues con placer escucharía a alguien el relato (lógoi) de las batallas en las que suele participar una ciudad que combate con otras ciudades, llega bien dispuesta a la guerra y, durante la lucha, hace lo que corresponde con su educación y formación (têi paideíai kaì trophêi) no sólo en la acción, sino también en los tratados con cada uno de los estados. (Tim. 19b3-c9)

En el resumen realizado por Sócrates y Timeo se trazan los lineamientos básicos para la educación y formación de la mejor instrucción política (áriste politeía), comparada posteriormente con un bellos animales (zôia kalá). A fin de ver su discurso estático, su conjunto de normas programáticas en movimiento (kinoúmena), esto es, desarrollando su 
existencia narrativamente, solicita un discurso (lógos). En el pedido de Sócrates el mito de la Atlántida no busca ningún tipo de narración histórica (en el sentido de describir fielmente una realidad material), sino que persigue encontrar un relato acorde al cuadro descrito, esto es -según marca Johansen- ${ }^{5}$ que tenga relación no con la verdad de lo acontecido, sino con las verdades filosóficas del ordenamiento político expuesto con anterioridad. Así, el mito de la Atlántida no es otra cosa que un mito en la forma que se reclama en República: una historia apropiada para educar mediante el ejemplo, para informar y establecer modos de conducta y pensamiento convenientes al conjunto de la sociedad.

Uso pedagógico
del mito por parte
de los
gobernantes.

"Lo que me sucede no es
nada extraño, pues tengo la
misma opinión que los
poetas (tôn poietôn)
antiguos y que los actuales
y, aunque no desdeño en
absoluto al linaje poético (tò
poietikòn génos), es
evidente que el pueblo de
los imitadores (tò mimetikòn
éthnos) imitará muy
fácilmente y de manera
sencilla y de la mejor
manera (mimésetai rhâista
kai árista) aquello en lo que
ha sido educado (tês
trophês). Sin embargo, a
cualquiera le resulta muy
difícil (khalepón) imitar bien
en obras (érgois) lo que está
fuera de su propia
educación y le es aún más
dificultoso (khalepóteron)
imitarlo bien (eû mimeîsthai)
en palabras (lógois)"
(Tim. 19d2-e2)

\author{
"-¿No entiendes-dije yo- que primero \\ les contamos mitos a los niños (hóti \\ prôton toîs paidíois mýthous \\ légomen)? En general son falsos \\ (pseûdos), aunque hay también en \\ ellos algo verdadero (alethê). Pero \\ para la educación (tà paidía) nos \\ valemos de los mitos (mýthois) antes \\ que de los ejercicios físicos. \\ [...] \\ - ¿Y no sabes que el origen es la parte \\ más importante (mégiston) de toda \\ tarea, especialmente para alguien \\ joven y delicado (néôi kaì hapalôi)? \\ Sin duda en ese momento se modela \\ (pláttetai) mejor e incorpora mejor el \\ modelo que se le quiere inculcar a \\ cada uno- \\ -Completamente-dijo. \\ -Entonces, ¿acaso permitiremos tan \\ fácilmente que los niños (toùs paîdas) \\ escuchen mitos (mýthous) \\ cualesquiera compuestos por el \\ primero que pase y que se introduzcan \\ en sus almas (lambánein en taîs \\ psykhaîs) opiniones (dóxas) que en la \\ mayoría de los casos se oponen
}




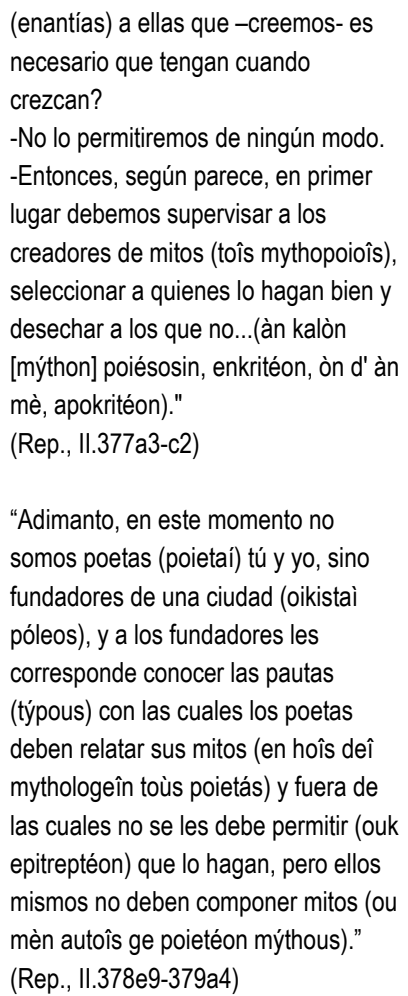

Los parágrafos seleccionados de República y de Timeo muestran el claro uso del mito para formar una educación (paideía), utilizándolo para modelar (pláttetai) el alma (psykhén) cuando ésta se encuentra más predispuesta para ser formada, en la niñez (tà paidía) ${ }^{6}$. Los poetas (poietaí), según el programa platónico, deben redactar sus mitos (mýthoi) de acuerdo a los preceptos (týpoi) establecidos por los fundadores de la ciudad (oikistês)-filósofos, guiados por verdades filosóficas y no por opiniones falsas (pseudês dóxa) que pueden alterar la educación de los jóvenes y, consecuentemente, el funcionamiento de la ciudad (polis) ${ }^{7}$. Esta primera

6 Para la relación entre el mito de la Atlántida y República en lo que respecta a educación ver (MORGAN, 1998), y (LAPLACE, 1984). Para el uso conciente del mito por parte de los guardianes y su lugar en República y Leyes ver (SOARES, 2004).

7 Sobre la censura hecha a los poetas por parte del gobierno ver (BALOT, 2006, p. 202 y ss.). 
diagramación de los mitos está pensada en función de reproducir en el común de la sociedad, tanto en la palabra (lógos) como en los actos (érga), el esquema ético dispuesto.

\section{IV}

En resumen, hemos visto las similitudes entre pasajes seleccionados de República y de Timeo, buscando una nueva orientación para comprenderlas. Denominamos a este renovado enfoque estadio basal del pensamiento político platónico. Con este concepto hacemos referencia a una base de diagramaciones político-sociales de largo plazo en el pensamiento de Platón, presentes tanto en República como en Timeo.

Los indicios de una permanencia de ciertos elementos teóricos, de este estadio basal, abona el terreno para una superación de los modelos extremos de lectura evolutiva que corrían el riesgo de convertir los distintos períodos de la producción platónica en ámbitos inconmensurables. El estadio basal del pensamiento político platónico, por el contrario, encarna un complejo de tesis de cierta estabilidad que resisten las variaciones epocales. Por esta vía los desarrollos político-sociales de los libros iniciales de República dejan de ser un muestreo de propuestas restringidas a la madurez, para instalarse como un registro particular de un núcleo de posiciones que se manifiesta igualmente en obras de otros períodos, como muestra el Timeo y sanciona, por tanto, un proyecto de largo plazo. Sin necesidad de volver a los modelos puros de lectura integral, el estudio del estadio basal se integra a las líneas más recientes que prestan atención a la dimensión dramatológica como elemento para superar las limitaciones del evolutivismo y avanzar en un enfoque menos disruptivo de la producción platónica ${ }^{8}$.

8 La propuesta del presente trabajo, el concepto de estadio basal del pensamiento político platónico, se integra a una investigación más amplia. Como se ha indicado en las notas 7, 8 y 9, es central revisar textos que se encuentran realizando claras alusiones intertextuales a los primeros libros de República 0 , bajo nuestra línea interpretativa, a propuestas del estadio basal. Este conjunto de fuentes comprende obras completas o pasajes de Jenofonte, Aristófanes, Aristipo, Antístenes e Isócrates. Otro elemento clave a la hora de ampliar el análisis del estadio basal es el análisis de otras obras del corpus Platonicum que tocan temáticas políticas. Más allá de que, efectivamente, las coincidencias entre República y Timeo sean suficiente prueba de un número de propuestas de largo plazo en el pensamiento platónico, creemos que es posible encontrar elementos adicionales que atestigüen estas pervivencias, extendiendo su período de vigencia. Finalmente, consideramos el estadio basal un 


\section{Referências:}

\section{Texto Griego:}

ADAM, J. (ed.) The Republic of Plato, Cambridge: Cambridge University Press, 1902.

BURNET, I. (ed.) Platonis Opera, London: Oxford Univesity Press, 1900-1907.

CAMPBELl, L., JOWETT, B., (ed. y trad.), Plato's Republic, Cambridge: Cambridge University Press, 1984.

CORNFORD, F. (ed. y trad.), Plato's Cosmology, The Timaeus of Plato, Indianapolis: Hackett Publishing Company, 1997.

LIVINGSTONE, N. (ed.), A commentary on Isócrates’ Busiris, Menemosyne Supplement 223, Leiden: Brill, 2001.

SHOREY, P. (ed. y trad.), The Republic of Plato, Oxford: Oxford University Press, 1937.

TAYLOR, A.E. (ed.), A Commentary on Plato's Timaeus, London: Oxford University Press, 1927.

\section{Texto Latino:}

ROLFE, J. (ed. y trad.), The Attic Nights of Aulus Gellius, London: Harvard University Press, 1927.

\section{Traducciones:}

BLOOM, A, (trad.). The Republic of Plato, New York: Basic Books, 1968.

CAMPBELL, M.A., JOWETT, B., (ed. y trad.), Plato's Republic, Cambridge: Cambridge University Press, 1894.

CORNFORD, F. (ed. y trad.), Plato's Cosmology, The Timaeus of Plato, Indianapolis: Hackett Publishing Company, 1997.

MÁRSICO, C., DIVENOSA, M., (trad.), Platón, República, Buenos Aires: Losada, 2005.

elemento de particular interés para la lectura de los pasajes de Política de Aristóteles en donde las teorizaciones políticas de Platón son criticadas: en una primera lectura que necesita ser ampliada por un trabajo en profundidad, se nos presenta sugerente comprender una referencia, por parte del Estagirita, a este conjunto de propuestas políticas que denominamos estadio basal del pensamiento político platónico. 
LAMB, W.R.M (trad.), Plato. Plato in Twelve Volumes, Vol. 9, London: Harvard University Press, 1925.

LISI, F., (trad.), Platón, Diálogos VI: Filebo, Timeo, Critias, Madrid: Gredos, 1992. ROLFE, J. (ed. y trad.), The Attic Nights of Aulus Gellius, London: Harvard University Press, 1927.

SHOREY, P. (ed. y trad.), The Republic of Plato, Oxford: Oxford University Press, 1937.

\section{Bibliografía secundaria:}

BALOT, R. Greek Political Thought, UK: Blakcwell Publishing, 2006.

BARKER, E. Greek Political Theory, Plato and his Predecesors, London : Methuen, 1918.

BRANDWOOD, L. The Chronology of Plato's Dialogues, Cambridge: Cambridge University Press, 1990.

CAMPBELL, M.A. The Sophistes and the Politicus of Plato, London: Clarendon Press, 1867.

FORDE, S. Gender and Justice in Plato. The American Political Science Review, Vol. 91, No. 3, 1997, pp. 657-670.

FRIEDLÄNDER, P. Plato, in 3 vols., New York: Pantheon Books, 1958-1969, p. 50 y ss

GIANNANTONI, Socratis et Socraticorum Reliquiae, Napoli: Bibliopolis, 1990.

GILL, C. The Genre of the Atlantis Story. Classical Philology, Vol. 72, No. 4, 1977, pp.287-304.

HOWLAND, J. Re-reading Plato: The Problem of Platónic Chronology. Phoenix, Vol. 45, No. 3, 1991, p. 189-214

HOWLAND, J., Xenophon's Philosophic Odyssey: On the Anabasis and Plato's Republic. The American Political Science Review, Vol. 94, No 4, 2000.

. The Republic: The Odyssey of Philosophy, USA: Paul Dry Books, 2004.

ILLARRAGA, R., "Primeras aproximaciones al estudio comparado de las propuestas políticas utópicas en la Ilustración Helena: conexiones entre el Busiris de Isócrates y los libros II y V de República" en A.A.V.V., Actas de las V Jornadas Nacionales de Pensamiento Clásico, UNRosario, Rosario, 2009.

JOHANSEN, T. Plato's Natural Philosophy, A Study of the Timeaus-Critias, New York: Cambridge University Press, 2004, pp. 24-47.

KAHN, C. Proleptic Composition in the Republic, o why Book 1 Was Neves a Separate Dialogue. The Classical Quarterly, Vol. 43, Vol. 1, p.131-142, 1993. 
MÁRSICO, C. Zonas de tensión dialógica. Perspectivas para la didáctica de la filosofía antigua. Buenos Aires: Ediciones del Zorzal, 2010.

MOORS, K. The Argument Against a Dramatic Date for Plato's Republic. Polis, Vol,7, No. 1, 1987, p. 6-31.

MORGAN, K. Designer History: Plato's Atlantis Story and Fourth-Century Ideology. The Journal of Hellenic Studies, Vol. 118, 1998, pp. 101-118.

NAILS, D. Agora, Academy, and the Conduct of Philosophy, Dordrecht: Kluwer Academic Publishers, 1995.

. The Dramatic Date of Plato's Republic. The Classical Journal, Vol. 93,

No. 4, p. 383-396, 1998

. The People of Plato: A Prosopography of Plato and Other Socratics, Hackett: Indianapolis, 2002.

NADON, C., From Republic to Empire: Political Revolution and the Common Good in Xenophon's Education of Cyrus. The American Political Science Review, Vol. 90, No.2, pp. 361- 374, 1996.

LAPLACE, M. Le "Critias" de Platon, ou l'ellpise d'une epopee, Hermes, Vol. 112, No. 3, 1984, pp. 377-382.

LIVINGSTONE, N. (ed.) A commentary on Isócrates' Busiris, Menemosyne Supplement 223, Leiden: Brill, 2001.

LAMPERT, L., PLANEAUX, C. Who's Who in Plato's Timaeus-Critias and Why. The Review of Metaphysics, Vol. 52, No. 1, p. 87-125, 1998, pp. 93-95.

LEDGER, G.R. Re-counting Plato: A Computer Analysis of Plato's Style, Oxford: Clarendon Press, 1989.

LUTOSLAWSKI, W. The origin and growth of Plato's logic, London, New York, Bombay: Longmans, Green and Co., 1897.

OKIN, S. Philosopher Queens and Private Wives: Plato on Women and the Family. Philosophy and Public Affairs, Vol. 6, No. 4, 1977, pp. 345-369.

POST, L.A. An Attemp to Reconstruct the First Edition of Plato's Republic. The Classical Weekly, Vol. 21, No. 6, p. 41-44, 1927.

REEVE, C.D.C. Philosopher-Kings: The Argument of Plato's 'Republic', Indianapolis: Hackett, 2006, pp. 22-24.

SALKEVER, S.G. Women, Soldiers, Citizens: Plato \& Aristotle on the Politics of Virility. Polity, Vol. 19, No. 2, 1986, pp. 232-253.

SAXONHOUSE, A. W. The Philosopher and the Female in the Political Thought of Plato. Political Theory, Vol. 4, No. 2, pp. 195-212.

SEGAL, E. Oxford Readings in Aristophanes, Oxford-New York: Oxford, 1996. 
SCHOFIELD, M. Saving the City, Philosopher-Kings and Other Classical Paradigms, London:Routldge, 1999.

. Approaching the Republic, en ROWE, C., SCHOFIELD, M. (eds.), The

Cambridge History of Greek and Roman Political Thought, New York: Cambridge, 2008.

TAYLOR, A.E. Plato, the Man and his Work, London: Methuen, 1955.

THESLEFF, H. Studies in Platónic Chronology, Helsinki: Societas Scientiarum Fennica, 1982.

THESLEFF, H. Platonic Chronology. Phronesis, Vol. 34, No. 1, p.1-26, 1989.

. The Early Version of Plato's Republic. Arctos, Vol. 31, p.149-74, 1997.

Email: rodrigoillarraga@gmail.com

Recebido em: Abril de 2011

Aprovado em: Maio de 2011 